Service social

\title{
Le pouvoir dans la réalisation des politiques sociales
}

\section{Vincent Lemieux}

Volume 38, numéro 2-3, 1989

Les politiques sociales

URI : https://id.erudit.org/iderudit/706437ar

DOI : https://doi.org/10.7202/706437ar

Aller au sommaire du numéro

Éditeur(s)

École de service social de l'Université Laval

ISSN

1708-1734 (numérique)

Découvrir la revue

Citer cet article

Lemieux, V. (1989). Le pouvoir dans la réalisation des politiques sociales.

Service social, 38(2-3), 179-195. https://doi.org/10.7202/706437ar

\section{Résumé de l'article}

Le projet de l'auteur est ici de circonscrire le secteur des politiques sociales à l'intérieur du domaine des politiques publiques, pour ensuite proposer une définition du pouvoir, une typologie des acteurs politiques et une présentation des principales alliances qui existent actuellement dans le secteur de la santé et des services sociaux. d'utilisation que vous pouvez consulter en ligne.

https://apropos.erudit.org/fr/usagers/politique-dutilisation/ 
Lemieux, Vincent, professeur au

Département de science politique,

Université Laval.

\section{Le pouvoir dans la réalisation des politiques sociales}

\section{Vincent Lemieux*}

Comment analyser les politiques sociales de façon à être capable d'agir sur elles, au besoin? Nous allons proposer que les phénomènes de pouvoir doivent être au cœur d'une telle démarche analytique, parce que la réalisation ou la non-réalisation des politiques publiques dépend de l'exercice du pouvoir.

Comme l'a écrit un des meilleurs spécialistes du pouvoir, Robert Dahl (1973: 49) :

"L'analyse du "pouvoir" n'est pas une simple entreprise de théorisation, mais un travail d'une haute importance pratique. Car notre façon d'agir dans la vie politique dépend largement de nos croyances à l'égard de la nature, de la distribution, et des pratiques du " pouvoir " qui caractérisent le système politique auquel nous sommes confrontés. Si l'on agit en croyant que le pouvoir est plutôt largement réparti au sein de la communauté, alors qu'en fait il est hautement concentré, ou si l'on agit en supposant que le pouvoir est entre les mains de quelques-uns alors qu'en réalité il est réparti entre une grande variété d'individus et de groupes qui doivent négocier, marchander, convaincre et séduire afin d'atteindre leurs buts, alors on risque de commettre de sérieuses erreurs politiques (...) Rien n'est plus néfaste pour la stratégie politique qu'une insuffisante compréhension du "pouvoir ", qu'une ignorance de la "structure du pouvoir", car se tromper sur le "pouvoir " c'est s'induire en erreur sur les perspectives d'avenir et les moyens de la stabilité, du changement et de la révolution. Les cimetières de I'histoire sont remplis de réformateurs ratés, de révolutionnaires qui, soit ne prirent jamais le pouvoir, soit, l'ayant pris, ne réalisèrent pas leurs objectifs, de contre-révolutionnaires incapables précisément de contrer les révolutions - des hommes (et des femmes) qui échouè- 
rent non seulement à cause du rapport des forces, mais surtout parce que leurs conceptions du pouvoir et de l'influence étaient à la fois simplistes et inexactes. »

Dans cet article nous voudrions d'abord circonscrire le secteur des politiques sociales à l'intérieur du domaine des politiques publiques, pour ensuite proposer une définition du pouvoir, une typologie des acteurs politiques et une présentation des principales alliances qui existent actuellement dans le secteur de la santé et des services sociaux. Les politiques concernant les Centres locaux de services communautaires (C.L.S.C.) serviront à illustrer les jeux de pouvoir des acteurs et de leurs alliances. En conclusion nous ferons quelques considérations pratiques sur l'exercice du pouvoir dans la réalisation des politiques de la santé et des services sociaux.

\section{Les politiques sociales}

II est utile de concevoir des politiques publiques en termes de régulation. Des moyens d'action, leur distribution, des relations entre les acteurs font problème aux yeux de certains participants à la réalisation des politiques. Ils cherchent, par l'exercice du pouvoir, à les ramener à une norme dont ils se sont éloignés ou à les amener à une norme qui n'a pas encore été atteinte. D'autres participants ne voient pas de problèmes ou voient d'autres problèmes dans les mêmes situations, et cherchent eux aussi à exercer du pouvoir, selon des préférences différentes. Des alliances, explicites ou implicites, se dessinent entre les acteurs qui ont des préférences voisines les unes des autres. Elles s'opposent à d'autres alliances, dont les préférences sont plus éloignées des leurs.

Quant à leurs moyens d'action, les acteurs sociaux sont définis ici comme :

- des personnes (moyens humains)

- finalisées (moyens normatifs)

- en des postes (moyens positionnels)

- d'où elles commandent (moyens actionneurs)

- l'usage de ressources matérielles (moyens matériels)

- ou informationnelles (moyens informationnels).

Les différents secteurs des politiques publiques peuvent être définis par des ensembles particuliers de ces moyens d'action, généralement centrés sur l'un ou quelques-uns d'entre eux. Ainsi les politiques économiques visent des ensembles centrés sur les moyens matériels, les politiques culturelles visent des ensembles centrés sur 
les moyens informationnels mais aussi sur les moyens normatifs, les politiques de réglementation visent des ensembles où les postes et les commandes sont centraux, etc.

De ce point de vue, les politiques sociales se caractérisent en ce qu'elles sont centrées sur ces moyens d'action très particuliers que sont les personnes. Ces moyens sont le support de tous les autres moyens, comme l'indique d'ailleurs la définition des acteurs sociaux que nous avons donnée plus haut. Les problèmes qui sont à l'origine des politiques sociales tiennent, dans une perspective individualiste, à des dysfonctions des personnes, qui diminuent leur aptitude à se finaliser, à occuper correctement leurs postes, à exercer des commandes et à user de ressources matérielles ou informationnelles.

Dans une perspective plus sociale, c'est plutôt le manque de moyens normatifs, positionnels, actionneurs, matériels ou informationnels qui entraîne des dysfonctions chez les personnes en tant que supports de tous les autres moyens. Quoi qu'il en soit, les personnes sont les centres déterminants ou déterminés des ensembles de moyens d'action que visent les politiques sociales. On peut interpréter ainsi les politiques dans le secteur de la santé, des services sociaux, de la main-d'œuvre, de la famille, de la vieillesse, etc.

\section{Le pouvoir}

La réalisation ou la non-réalisation des politiques publiques, à travers les différents sous-systèmes de la régulation, repose sur l'exercice du pouvoir. C'est du moins la perspective que nous adoptons, aux fins de cet article (sur ce point, voir Lemieux, 1986).

Le pouvoir, c'est de transformer un choix en une décision. De façon plus explicite, nous dirons que le pouvoir, pour un acteur social, consiste à contrôler selon ses préférences les décisions qui concernent ses moyens d'action ou ceux d'autres acteurs sociaux. Les moyens d'action sont les enjeux du pouvoir mais en sont aussi les atouts. Un travailleur social exerce du pouvoir quand, suite à une intervention de sa part, des normes existantes dans son milieu de travail sont modifiées. II n'exerce pas de pouvoir quand il échoue dans sa tentative de convaincre un bénéficiaire de cesser de consommer de l'alcool ou de la drogue. Dans le premier cas, des moyens normatifs sont l'enjeu du pouvoir, dans le second cas, il y a aussi des moyens matériels. Quant aux atouts du travailleur social, ce sont ses propres normes, le poste qu'il occupe, son information, etc.

Chacun des trois grands sous-systèmes fonctionnels par lesquels se réalisent les politiques publiques donne lieu à des relations de pouvoir. En voici quelques exemples : 
- dans le sous-sytème de prise en charge des problèmes, certains acteurs arrivent à agir sur les commandes du gouvernement ou de l'adrninistration compétente, pour qu'un problème soit mis à l'ordre du jour, alors que d'autres acteurs n'y arrivent pas (sur la mise à l'ordre du jour, voir Kingdon, 1984).

- dans le sous-système d'élaboration des solutions, le parti ministériel réussit à faire adopter une loi qui affecte les moyens d'action des acteurs concernés, contre le parti d'opposition impuissant dans ses tentatives visant à modifier le projet de loi.

- dans le sous-système de la mise en cuvre des règles, entendues au sens large, des fonctionnaires choisissent sciemment $d$ 'ignorer telle ou telle règle, ou de la contourner, alors que d'autres sont manipulés par des clients qui ont de bons " contacts » politiques (sur I'application des politiques, voir entre autres Bardach, 1977).

\section{Les acteurs politiques}

Les acteurs sociaux deviennent des acteurs politiques quand ils participent de quelque façon aux relations de pouvoir par lesquelles se réalisent les politiques publiques.

De par les postes qu'ils occupent dans une organisation et de par leur compétence spécialisée ou non dans les décisions par lesquelles se réalisent les politiques publiques, les acteurs politiques appartiennent, dans le secteur des politiques sociales comme dans les autres secteurs, à quatre grandes catégories : les responsables, les agents, les intéressés et les populations. Ces catégories ne sont pas exclusives (les populations comprennent les trois autres catégories, etc.) Elles ne sont pas des catégories de personnes, mais d'acteurs, c'est-à-dire de personnes en des postes sociaux.

Les responsables occupent les postes de direction dans le système de gouverne d'une organisation. Ils sont généralement choisis par les populations ou par des groupes d'intéressés. Ils n'ont pas une compétence spécialisée mais générale en matière de politiques publiques, du moins au palier où ils se trouvent. Dans le secteur des politiques de santé et de services sociaux, les responsables sont, au palier du ministère, le conseil de ministre, le ministre et son cabinet politique. Au palier des conseils régionaux de la santé et des services sociaux (C.R.S.S.S.), ce sont les membres du conseil d'administration. Il en est de même au palier des C.L.S.C. 
Les agents occupent eux aussi des postes officiels dans le système de gouverne d'une organisation. Les principaux d'entre eux tout au moins sont généralement nommés par les responsables. Ils ont une compétence spécialisée en matière de politiques publiques, bien qu'il arrive que certains d'entre eux soient aussi des généralistes (on pense par exemple à un sous-ministre en titre ou à un directeur général d'établissement). À chacun des paliers, ministère, conseils régionaux ou conseils des services sociaux, centres locaux de services communautaires, les agents se subdivisent en au moins trois grandes catégories : cadres, professionnels et employés de soutien.

Les intéressés, au sens où nous l'entendons, n'appartiennent pas au système de gouverne. On les trouve plutôt dans les publics d'une organisation. Comme les responsables et les agents, ils sont le plus souvent rassemblés en des groupes, bien qu'il puisse arriver à la limite qu'un individu seul agisse comme intéressé (par exemple, une personne qui s'exprime via le courrier du lecteur d'un quotidien). Dans le secteur des politiques de la santé et des services sociaux, les groupes d'intéressés sont très nombreux, et cela aux trois paliers que nous distinguons : central, régional et local. Ils vont des puissantes associations de médecins aux groupes communautaires en passant par les médias et les experts universitaires ou autres, qui sont eux aussi des intéressés. Ces intéressés sont spécialisés dans certaines décisions qui les "intéressent» plus que d'autres. L'exemple des associations de médecins montre que des personnes qui sont à certains égards des agents (dans les centres hospitaliers, entre autres) peuvent se rassembler à d'autres égards en des groupes d'intéressés, qui se définissent alors comme des publics d'une politique plutôt que comme ses agents. Ajoutons qu'il ne faut pas confondre groupe d'intéressés et groupe de pression : les agents peuvent fort bien agir, à titre d'agents, comme groupe de pression en vue de la réalisation ou non d'une politique, sans être pour autant des intéressés au sens où nous l'entendons.

Les populations se distinguent des autres acteurs politiques en ce qu'elles sont des agrégats, ou des groupes statistiques (qu'on nomme parfois groupes tertiaires) et non des groupes organisés. Ce sont, par exemple, les électorats ou encore les bénéficiaires d'une politique ou d'un programme, considérés comme un ensemble d'individus. De ce point de vue, les populations n'appartiennent pas au système de gouverne et elles n'ont pas de compétence spécialisée dans des décisions reliées aux politiques publiques. Elles peuvent quand même exercer du pouvoir à l'occasion des élections, ou encore par les résultats de sondages ou d'autres données, quand elles influencent 
des décisions contrôlées apparemment par d'autres acteurs politiques.

\section{Les alliances entre les acteurs}

Les acteurs qui participent à la réalisation des politiques de santé et de services sociaux sont très nombreux. Au Québec, il y a deux partis qui regroupent les responsables du palier central et leurs supporters, le Parti libéral et le Parti québécois. Des responsables au palier régional et au palier local appartiennent à ces partis, mais la plupart d'entre eux ont leurs principales allégeances à d'autres groupes ou organisations. Les agents sont très nombreux, à tous les paliers, et leurs préférences quant aux politiques publiques varient selon les directions où ils se trouvent au ministère et selon les organisations où ils travaillent dans le réseau des services de santé et des services sociaux. Les groupes d'intéressés sont encore plus divers : les principaux regroupent des établissements, des professionnels et des employés de soutien, des bénéficiaires ou des acteurs dans la communauté, en des groupes qu'on nomme justement communautaires. Il y a aussi les populations considérées dans leur aspect territorial ou encore dans leur aspect plus fonctionnel, en termes de clientèles (personnes âgées, personnes en perte d'autonomie, jeunes délinquants, malades mentaux, etc.)

Parmi ces multiples participants, certains agissent de façon convergente ou encore forment des coalitions pour augmenter leurs chances d'exercer du pouvoir, c'est-à-dire de contrôler des décisions qui affectent leurs moyens d'action ou encore ceux des autres acteurs ou coalitions qui leur sont opposés ou par rapport auxquels ils sont ambivalents.

Quand on examine l'évolution du secteur de la santé et des services sociaux depuis une vingtaine d'années, c'est-à-dire depuis la création du ministère des Affaires sociales et l'adoption, en 1971, de la loi sur l'organisation des services de santé et des services sociaux, on arrive à identifier un certain nombre d'alliances qui ont exercé à un moment ou l'autre, seules ou avec l'aide d'autres alliances, un pouvoir important dans la réalisation ou non des politiques publiques. Nous parlons d'alliances en y englobant à la fois des coalitions explicites et des convergences plus implicites dans le contrôle des décisions publiques.

En simplifiant beaucoup, on peut ramener ces alliances à six, qui n'ont pas toujours des frontières précises et dont certaines se recoupent en partie, mais qu'on peut caractériser par des valeurs différentes qui guident leur lecture des problèmes sociaux, des solutions à adopter et des règles à appliquer. 
Il y a d'abord les deux grandes alliances partisanes, celle du Parti libéral et celle du Parti québécois. Les principales oppositions entre elles ne se manifestent pas dans le secteur de la santé et des services sociaux. Quand les deux s'opposent, le Parti libéral a sur les personnes des normes plutôt individuelles, alors que le Parti québécois a des normes plus sociales, pour reprendre la distinction que nous faisions au début, à propos de la spécificité des politiques sociales. Les deux alliances partisanes ont surtout ceci de commun qu'elles voient les problèmes, les solutions et les règles dans une perspective électorale et donc compétitive, en accordant une attention spéciale aux opinions et comportements des populations.

Les deux alliances partisanes, qui ont participé à la direction du gouvernement durant un nombre à peu près égal d'années entre 1970 et aujourd'hui, sont proches de la grande alliance bureaucratique qui rassemble beaucoup d'agents du ministère, des conseils régionaux et des autres établissements du réseau. Pour cette alliance complexe, où l'on peut sans doute distinguer des sous-alliances, tous les enjeux sont importants, et même si le sous-système de la mise en œuvre des règles est celui qui la préoccupe le plus, ses imposants moyens d'action l'amènent à exercer un pouvoir certain dans le sous-système de la prise en charge et dans celui de l'élaboration également. On peut considérer que cette alliance s'étend à des milieux universitaires, en particulier à ceux qui ont collaboré aux travaux de la commission Rochon et à ceux de la commission CastonguayNepveu avant elle (sur ce point voir Lemieux, 1989; Bergeron et Grégoire, 1988).

Non moins puissante est l'alliance médicale, entendue au sens de la " médicalisation » de la santé. Elle regroupe des agents et des intéressés qui disposent d'un peu tous les atouts du pouvoir, mais dont le principal, qui fonde tous les autres, est d'ordre technologique, avec le mélange de normatif, d'informationnel et de matériel que cela suppose. Pour les professionnels et les gestionnaires inclus à l'intérieur de cette alliance, la vision des problèmes, des solutions et des règles qui composent les politiques publiques est plus individuelle que sociale, et la matérialisation des soins et services, par le développement de la technologie, est un des enjeux principaux des politiques publiques. Même si les trois alliances précédentes s'opposent parfois à l'alliance médicale, les jeux de coopération avec elle sont plus déterminants que les jeux de conflit (sur ce point, voir Tuohy, 1988). 
L'alliance qu'on peut dire socio-sanitaire, ou encore sociocommunautaire, pour indiquer sa valorisation de la dimension sociale de la santé entendue au sens large, regroupe les agents, les intéressés et les populations qui sont opposés à la domination excessive à leurs yeux de l'alliance médicale. On retrouve en particulier dans cette alliance les médecins de santé communautaire et la plupart des travailleurs sociaux. Les atouts normatifs, informationnels et matériels de cette alliance sont inférieurs à ceux de I'alliance bureaucratique et de I'alliance médicale. Par contre l'alliance socio-sanitaire est riche en ressources humaines, surtout quand on considère les nombreux groupes communautaires de services ou d'entraide qui en font partie.

Enfin, il y a émergence depuis quelques années d'une nouvelle alliance, encore en état de formation, et qui se recrute en bonne partie parmi les autres. Cette alliance peut être dite managériale, ou encore entrepreneuriale, pour indiquer la distance qu'elle prend par rapport à l'alliance bureaucratique. On y retrouve des responsables du Parti libéral, des agents qui sont dans des postes de direction au ministère et dans des établissements du réseau, des experts universitaires et d'autres intéressés. Pour les membres de cette alliance, les ressources humaines, qui sont au centre de l'ensemble des moyens spécifiques aux politiques sociales, doivent être gérées comme les autres moyens d'action, de façon efficiente et efficace. Il faut pour cela introduire de la compétition dans un système de régulation qui est trop monopoliste, cette compétition devant passer par la privatisation entendue au sens strict, et aussi au sens plus large d'une introduction dans le secteur public des méthodes de gestion du secteur privé (sur ce point, voir Ouellet, 1987).

Ces six alliances se recoupent entre elles et il y a des passages de l'une à l'autre. Les rapports entre les alliances sont aussi plus ou moins proches ou distants (voir le tableau 1). On peut distinguer quatre degrés à cet égard : des rapports proches $(+)$, plus proches que distants $( \pm)$, plus distants que proches $(\mp)$, et distants $(-)$. II a évidemment été posé qu'une alliance a un rapport proche avec ellemême. Par contre elle n'a pas de rapport proche avec une autre, car les deux alliances devraient alors être ramenées à une seule. 


\section{TABLEAU 1}

La proximité et la distance entre les six alliances dans le secteur de la santé et des services sociaux

\begin{tabular}{|lcccccc|}
\hline & MA & ME & PL & BU & PQ & SS \\
\hline MA & + & \pm & \pm & \pm & $\mp$ & - \\
ME & \pm & + & \pm & \pm & $\mp$ & - \\
PL & \pm & \pm & + & \pm & $\mp$ & $\mp$ \\
BU & \pm & \pm & \pm & + & $\mp$ & $\mp$ \\
PQ & $\mp$ & $\mp$ & $\mp$ & $\mp$ & + & \pm \\
SS & - & - & $\mp$ & $\mp$ & \pm & + \\
MA : alliance managériale & BU : alliance bureaucratique \\
ME : alliance médicale & PQ : alliance péquiste \\
PL : alliance libérale & & SS : alliance socio-sanitaire \\
\hline
\end{tabular}

Le tableau appelle plusieurs commentaires :

$1^{\circ} \quad$ Les alliances sont disposées dans un ordre de "voisinage ». On pourra vérifier que deux alliances voisines dans le tableau ont autant ou plus de signes communs entre elles qu'avec les alliances dont elles ne sont pas voisines. On notera aussi que $d^{\prime}$ 'une voisine à l'autre, la différence entre les signes d'une même colonne n'est jamais de plus d'un degré.

$2^{\circ} \quad$ Les deux alliances qui sont les plus proches de l'ensemble des autres sont l'alliance libérale et l'alliance bureaucratique, qui sont d'ailleurs voisines l'une de l'autre. En fait les quatre premières alliances du tableau ont plusieurs signes en commun. L'alliance péquiste se démarque des quatre autres, mais par rapport à l'alliance bureaucratique, sa voisine, les différences de signe sont toutes d'un degré seulement. L'alliance socio-sanitaire, par contre, est la plus éloignée de l'ensemble des autres. Elle n'a que deux traits en commun avec les autres alliances : ses rapports plus distants que proches avec l'alliance libérale et l'alliance bureaucratique respectivement, qu'elle partage avec l'alliance péquiste. 
$3^{\circ} \quad$ Dans le cas des alliances partisanes, les rapports avec les autres alliances sont susceptibles de varier selon que le parti est au gouvernement ou dans l'opposition. Ainsi, au début des années 1980, I'alliance péquiste avait, avec I'alliance bureaucratique, des rapports plus proches que maintenant. Inversement, I'alliance libérale avait des rapports plus distants que maintenant avec cette alliance bureaucratique.

$4^{\circ}$ Ajoutons que la formation ou la disparition d'une alliance ne manque pas de transformer les rapports entre les autres alliances. La formation de l'alliance dite managériale a, par exemple, relâché les liens entre l'alliance libérale et l'alliance bureaucratique; elle pourra, si elle s'impose, resserrer les liens de cette alliance bureaucratique avec l'alliance socio-sanitaire, etc.

$5^{\circ} \quad$ Enfin, notons sur un plan plus théorique que la structure des rapports du tableau 1 est équilibrée (ou plus exactement semiéquilibrée) au sens de la théorie des graphes, en ce que dans chacune des vingt triades du système, si on s'en tient au signe dominant (le + dans le degré \pm , et le - dans le degré $\mp$ ), les rapports entre les alliances obéissent dans chacune des triades au principe structurel voulant que a) l'ami de mon ami est mon ami, b) l'ennemi de mon ami est mon ennemi, c) l'ami de mon ennemi est mon ennemi, et d) l'ennemi de mon ennemi est mon ami (sur ce point, voir Cartwright et Harary, 1979). Ainsi pour se limiter à la première triade, regroupant les alliances managériale, médicale et libérale, les trois alliances sont plutôt " amies » entre elles (les degrés sont plus proches que distants dans les trois cas), ce qui correspond à la première modalité du principe structurel énoncé plus haut.

Pour un acteur politique donné, le pouvoir qu'il exerce dans la réalisation des politiques publiques dépend de l'alliance ou des alliances auxquelles il se rallie et des rapports qui s'établissent avec les autres alliances. Nous voudrions le montrer en reconstituant en ces termes les politiques concernant les C.L.S.C. au cours des années 1970 et 1980 .

\section{Les politiques concernant les C.L.S.C.}

Dans la première version du projet de loi sur l'organisation des services de santé et des services sociaux, le C.L.S.C. est défini ainsi :

"Un établissement qui, sur une base locale, assure à la communauté des services d'action sanitaire et sociale, reçoit les personnes qui requièrent pour elles ou pour leurs familles des services de santé et des services sociaux courants, les conseille ou les dirige vers les 
autres institutions les plus aptes à leur venir en aide et, si nécessaire, leur prodigue les services de santé et les services sociaux courants. »

Cette définition souligne la vocation des C.L.S.C., telle qu'on la pensait alors. Portes d'entrée sur le réseau, ils sont chargés de fournir les services de santé et les services sociaux de première ligne. Dans les débats en commission parlementaire, des représentants de l'alliance médicale remettent en question cette vocation. Le président de la Fédération des médecins spécialistes du Québec (F.M.S.Q.) déclare que les C.L.S.C. sont nécessaires en milieu urbain défavorisé et, de façon temporaire seulement, en milieu rural. Inquiets de l'action des premiers C.L.S.C., qui sont en voie d'implantation à ce moment, les porte-parole de l'alliance médicale demandent qu'un conseil des médecins et dentistes soit créé à l'intérieur des C.L.S.C., et que les conditions de rémunération de ces professionnels soient négociées sur le plan provincial.

De leur côté, des porte-parole de l'alliance socio-sanitaire, dont les dirigeants de la Confédération des syndicats nationaux, demandent que les représentants de la population soient majoritaires à l'intérieur des conseils d'administration (ils sont 5 sur 10, dans le projet de loi). Le ministre Castonguay qui, à ce moment, est à la fois le leader de l'alliance libérale et de I'alliance bureaucratique, soutient, appuyé par le Parti québécois, que la création des C.L.S.C. est nécessaire pour juguler la montée des coûts dans le système. II consent à la création d'un conseil des médecins et dentistes, augmente à la fois la représentation des professionnels et nonprofessionnels au conseil d'administration, tout en ajoutant deux représentants de la population, nommés par le gouvernement (sur cette étape, voir Lemieux et Labrie, 1979 : 153-157).

Comme on le voit, cinq des six alliances que nous avons distinguées prennent position dès ce premier débat sur les C.L.S.C. Il ne manque que l'alliance managériale, inexistante à ce moment-là. Dans la suite, les C.L.S.C. demeureront constamment opposés à l'alliance médicale et entretiendront des relations plus ambivalentes avec les deux alliances partisanes. Les relations avec les deux autres alliances, la bureaucratique et la socio-sanitaire, seront au cœur du dilemme posé aux responsables et autres acteurs politiques rattachés aux C.L.S.C., dans leur participation à la réalisation des politiques publiques. Les C.L.S.C. doivent-ils miser principalement sur I'alliance bureaucratique ou sur I'alliance socio-sanitaire? Autrement dit, se définissent-ils comme des agences d'abord redevables au centre bureaucratique qu'elles prolongent auprès des populations et des groupes d'intéressés qui en sont proches, ou se considèrent-ils plutôt comme redevables envers les populations et les groupes qui 
requièrent leurs services, que le centre bureaucratique approuve ou non les services demandés?

Dès 1974, lors de l'étude des crédits du ministère des Affaires sociales, en commission parlementaire, deux médecins, députés libéraux, s'en prennent aux C.L.S.C. contre le ministre Forget. En arrière-plan de cette attaque, il y a les récriminations de plusieurs députés et autres partisans libéraux qui reprochent à des animateurs des C.L.S.C. de servir la cause du Parti québécois, sous le couvert de I'action communautaire. Cette conjugaison des deux alliances dominantes, la médicale et la libérale, contre les C.L.S.C., amène le ministre à créer, au début de 1975, un comité d'enquête sur les C.L.S.C., l'équipe Bilan. Cela lui permet de calmer l'opposition venant de l'alliance libérale et de gagner du temps dans la révision de la politique touchant les C.L.S.C.

Les recommandations du rapport majoritaire de l'équipe Bilan vont dans le sens d'une "bureaucratisation " des C.L.S.C. : il ne devrait y avoir qu'un seul type de C.L.S.C., ceux-ci devraient se concentrer sur la fourniture de services et non sur la transformation du milieu, ils sont appelés à coexister avec les cliniques et les polycliniques qui se multiplient sur le territoire, les conseils régionaux devraient jouer un rôle accru dans l'implantation et la coordination des C.L.S.C. Cette dernière recommandation allait inspirer la décentralisation de la fin des années 1970 .

Le rapport minoritaire conçoit au contraire les C.L.S.C. dans I'optique de I'alliance socio-sanitaire et d'une responsabilité première envers la population. II peut y avoir plusieurs types de C.L.S.C., I'action communautaire est essentielle, I'autonomie des C.L.S.C. est nécessaire au maintien de leur action innovatrice.

Les règles officielles appliquées dans les politiques publiques touchant les C.L.S.C., du milieu des années 1970 à nos jours, manifestent la prépondérance de l'alliance médicale. L'alliance bureaucratique et les alliances partisanes sont plus souvent ses partenaires que ses adversaires. En témoignent : la prolifération des cliniques et polycliniques daris les années 1970 alors que le ministère retarde jusqu'à la fin des années 1980 l'implantation des derniers C.L.S.C.; I'obtention par la Fédération des médecins omnipraticiens du Québec (F.M.O.Q.) du privilège de négocier avec le gouvernement les conditions de travail des médecins des C.L.S.C.; la décentralisation, aux mains des C.R.S.S.S., de certaines activités concernant les ressources financières et humaines des C.L.S.C. ainsi que l'implantation des nouveaux C.L.S.C. Toutes ces politiques ont contribué à rendre les C.L.S.C. plus dépendants, officiellement, de I'alliance bureaucratique que de I'alliance socio-sanitaire. 
Sur le terrain cependant, la plupart des C.L.S.C. se sont ralliés, en pratique, à l'alliance socio-sanitaire. C'est ainsi qu'un directeur général de C.L.S.C. écrivait au début des années 1980 (Breton, 1980) :

"Lorsqu'on a créé les C.L.S.C., on a voulu en faire la porte d'entrée du réseau des Affaires sociales. Je ne surprendrai personne ici en disant que plusieurs C.L.S.C. ne sont pas seulement la porte d'entrée du réseau des Affaires sociales mais bien la porte d'entrée de tout le réseau gouvernemental pour le citoyen ordinaire du district du C.L.S.C. J'irai plus loin, je dirai même que les C.L.S.C. se sentent à l'étroit à l'intérieur du ministère des Affaires sociales.

\begin{abstract}
À l'étroit d'abord, parce que les C.L.S.C. ont été créés pour répondre aux besoins socio-sanitaires d'une population donnée et ce, à la fois dans une dimension de services courants et de prévention des problèmes. Si la mission de répondre aux services socio-sanitaires courants cadre généralement bien avec le réseau des Affaires sociales, il en est différemment de la vocation préventive de nos établissements. Cette dernière nous amène rapidement à intervenir dans toutes sortes de domaines souvent éloignés des préoccupations traditionnelles des Affaires sociales. »
\end{abstract}

Cette expérience a été celle de la plupart des C.L.S.C., d'autant plus que sur le plan strictement socio-sanitaire, d'autres organisations, hôpitaux, polycliniques, Centres de services sociaux (C.S.S.) ont fourni eux aussi des services de première ligne. Elle a trouvé son écho dans le mémoire que la Fédération des C.L.S.C. a présenté en 1986 à la commission Parizeau sur l'avenir des municipalités. Tenant compte que les territoires des C.L.S.C. correspondent à ceux des municipalités régionales de comté (M.R.C.), la municipalisation des C.L.S.C. est envisagée comme une solution possible, dans l'avenir, au problème qu'ont les C.L.S.C. à concilier leur dimension sociosanitaire, et plus largement communautaire, avec leur dimension bureaucratique. Ce n'est probablement pas la solution préférée par les agents qui, à l'intérieur de l'alliance bureaucratique, ont les C.L.S.C. comme clientèle, et qui se trouvent ainsi opposés à ceux dont la clientèle est plutôt médicale et hospitalière. Par contre les tenants de la nouvelle alliance managériale ont sans doute moins de réticences envers cette proposition.

Le rapport du comité Brunet, dit de réflexion et d'analyse des services dispensés par les C.L.S.C., qui est paru en 1987, va plutôt, quant à lui, dans le sens d'un ralliement à l'alliance bureaucratique, même s'il cherche à le concilier avec le rattachement à l'alliance sociosanitaire. Les conclusions du comité Brunet ont d'ailleurs été plus ou moins entérinées par la commission Rochon. Le programme de services médicaux et psychosociaux courants et le programme de maintien à domicile devraient se retrouver dans chacun des C.L.S.C. 
Quant au reste, la diversité, excessive selon le comité Brunet, dans les activités des C.L.S.C., devrait être ramenée à quatre programmes spécifiques structurés en fonction des groupes à risque, dont un pourra être déterminé au niveau local.

Les orientations proposées par la ministre, au début de 1989, cherchent à rapprocher les alliances autres que partisanes les unes des autres, en proposant aux établissements et aux autres acteurs du réseau, en particulier les C.L.S.C., de partager les valeurs de plus d'une alliance. Les C.L.S.C. continuent d'être soumis à I'alliance bureaucratique qui règle leurs activités, comme elle règle dans le détail plusieurs des réformes proposées dans le document. Ce règlement des activités s'inspire de certaines valeurs de l'alliance managériale. C'est le cas, par exemple, quand il est recommandé que les C.L.S.C. cessent de faire du neuf à cinq et se rendent plus disponibles à leurs clientèles, le soir et en fin de semaine. En proposant que les C.L.S.C. assurent des services médicaux actuellement offerts par les centres hospitaliers, on les rapproche de l'alliance médicale. La mesure visant à n'avoir qu'un conseil d'administration par territoire de C.L.S.C., qui aurait autorité sur divers établissements, va dans le même sens. La participation des C.L.S.C. à I'alliance socio-sanitaire n'est pas pour autant écartée. Il est affirmé, en particulier, dans le docurnent, que le rôle des C.L.S.C. dans les services de maintien à domicile est apppelé à s'accentuer.

Reste à voir si cette vision des rapports entre les alliances, qui seraient plus coopératifs que conflictuels, pourra se réaliser, sous l'autorité d'un ministère où les composantes partisane, managériale et bureaucratique seraient elles-mêmes réconciliées.

Cette brève histoire, un peu sélective, des politiques concernant les C.L.S.C. enseigne que, dans ce secteur comme en d'autres, les acteurs politiques, pour exercer du pouvoir, ont à choisir (quand ils le peuvent...) leurs alliances contre d'autres alliances, ou à neutraliser des alliances qui pourraient leur être opposées, ou encore à se poser comme arbitres ou comme promoteurs de rapports à changer entre les alliances. L'autonomie des acteurs en ces matières est toujours limitée, cependant, par le pouvoir exercé sur eux par d'autres acteurs, qu'ils appartiennent ou non à la même alliance qu'eux.

\section{Conclusion}

Le type d'analyse des politiques sociales qui a été proposé dans cet article insiste sur le pouvoir des différents acteurs politiques dans la réalisation des politiques sociales, sur les atouts qui sont utilisés dans l'exercice du pouvoir et surtout sur les alliances qui regroupent 
les acteurs et entre lesquelles se joue le sort des politiques sociales. Les politiques dans le secteur de la santé et des services sociaux, et plus particulièrement celles qui concernent les C.L.S.C., ont servi à illustrer notre propos.

Certaines conclusions pratiques se dégagent de ce type d'analyse. Nous les résumerions en quatre points :

1) Chacune des alliances actuelles a des atouts importants, mais d'autres lui manquent ou encore ceux qu'elle possède ne lui sont pas acquis une fois pour toutes. Les alliances partisanes, la libérale et la péquiste, ont des atouts décisifs quand elles dirigent le gouvernement, mais dans l'opposition elles sont des alliances généralement peu puissantes par rapport aux autres. L'alliance bureaucratique est celle qui dispose des meilleurs atouts informationnels et actionneurs (par ses commandes dans l'application des politiques), mais elle est loin d'être parfaitement unifiée et demeure soumise, officiellement, à l'alliance partisane qui dirige le gouvernement. L'alliance médicale est sans doute la plus puissante face aux enjeux qui l'intéressent, mais elle n'a pas, contrairement aux autres, des stratégies à propos de l'ensemble des politiques sociales. L'alliance socio-sanitaire a des préoccupations plus vastes et rassemble plus de ressources humaines, mais tous ses autres atouts sont inférieurs à ceux des alliances dominantes. Enfin l'alliance managériale ne compte encore qu'un petit nombre de personnes, même si elles sont en bonne position dans le système, et son autonomie par rapport aux alliances libérale et bureaucratique est encore limitée. Comme les alliances partisanes, I'alliance managériale a cependant l'avantage de déborder le secteur des politiques sociales, et de pouvoir prétendre y proposer des mesures qui tiennent compte de l'ensemble du secteur public.

2) Les limites de chacune des alliances font que l'une ou l'autre d'entre elles ne peut espérer prédominer dans la réalisation des politiques publiques sans l'appui d'une ou d'autres alliances. Ainsi l'alliance médicale ne peut contrôler les enjeux qui la concernent dans les politiques publiques sans l'appui de l'alliance partisane dominante et de I'alliance bureaucratique. De plus en plus elle devra compter aussi sur l'appui de l'alliance managériale. De même, l'alliance socio-sanitaire n'aurait sans doute pas pu sauver les C.L.S.C. sans I'appui de l'alliance bureaucratique et celui, tout limité soit-il, de l'une ou l'autre alliance partisane. L'alliance managériale ne pourra imposer ses vues que si elle arrive à se rallier un peu toutes les autres alliances, ou encore à les neutraliser. 
3) Le nombre et la configuration des alliances ne sont pas quelque chose d'arrêté une fois pour toutes, que ce soit dans le modèle du chercheur ou dans la pratique sur le terrain. II y aurait peut-être lieu d'ajouter une alliance syndicale, distincte de I'alliance sociosanitaire ou encore de l'alliance bureaucratique, bien qu'il nous a semblé que, dans les années récentes tout au moins, les syndicats n'avaient pas des préférences suffisamment démarquées sur les politiques sociales pour qu'ils soient "consacrés" en une alliance. L'alliance managériale est de formation récente et son évolution demeure incertaine. II n'est pas impossible qu'un nouveau parti politique soit formé d'ici quelques années, qui ait des préférences originales sur les politiques sociales.

4) Il s'agit peut-être là d'un préjugé de politologue, qui a beaucoup étudié les partis, mais les alliances partisanes, quand elles sont au gouvernement, disposent d'un pouvoir d'initiative et d'un pouvoir de veto qui leur permettent, quand elles le veulent, de prédominer sur toutes les autres. À la condition, bien sûr, comme nous l'avons posé au point 2, d'obtenir l'appui d'autres alliances, ce en quoi les politiciens sont généralement habiles. Les responsables qui sont à la tête des alliances partisanes ont aussi l'aptitude de changer les règles officielles du jeu et même la nature du jeu. Ce sont eux, par exemple, et eux seuls, qui pourraient décider qu'on doit aller vers la municipalisation de certains établissements du réseau de la santé et des services sociaux, dont les C.L.S.C. Ce sont eux également qui, par le soutien ou non qu'ils apporteront à l'alliance managériale, décideront de sa réussite ou de son échec à inscrire plus de concurrence dans le système.

Pour reprendre les propos de Dahl, cités au début de cet article, on a une compréhension insuffisante du pouvoir dans la réalisation des politiques sociales, si on ignore que les responsables et leurs alliances sont les seuls à disposer officiellement d'un pouvoir constitutif. Ce pouvoir leur permet de prédominer au besoin dans les processus les plus encadrants de la réalisation des politiques publiques.

\section{Note}

* L'auteur est membre du Groupe de recherche sur les interventions gouvernementales (GRIG) et du Réseau de recherche en santé de l'Université Laval. 


\section{Références}

BARDACH, E., The Implementation Game, Cambridge, Mass., The M.I.T. Press, 1977.

Bergeron, P. et L. GréGOIRE, "Pour une vision émancipatrice de la recherche sur I'organisation du système de santé au Québec ", communication présentée au Congrès de l'Association canadienne de santé publique, à Québec, le 5 juillet 1988.

BRETON, J.G., "La décentralisation et les C.L.S.C. ", communication présentée au colloque du C.R.S.S.S. de la région 03 sur la décentralisation administrative, le 17 juin 1980.

CARTWRIGHT, D. et F. Harary, "Balance and Clusterability : an Overview ", dans Holland, P.W. et S. Leinhardt, (dir)., Perspectives in Social Network Research, New York, Academic Press, 1979 : 25-50.

DAHL, R.A., L'Analyse politique contemporaine, Paris, Laffont, 1973.

KIngdon, J.W., Agendas, Alternatives and Public Policy, Toronto, Little, Brown, 1984.

Lemieux, V., Les politiques publiques et l'exercice du pouvoir, Laboratoire d'études politiques et administratives, Université Laval, Cahier 86-01, juin 1986.

LEMIEUX, V., "La commission Rochon et la réalisation des politiques publiques ", Administration Publique du Canada, vol. 32, n 1, 1989 : 261-273.

LemieuX, V. et P. Labrie, " Le système gouvernétique des C.L.S.C. ", Recherches Sociographiques, vol. 20, $\mathrm{n}^{\circ} 2,1979$ : 149-171.

OUellet, L., "La privatisation : un instrument de management public ? ", Administration Publique du Canada, vol. $30, \mathrm{n}^{\circ} 4,1987$ : 566-584.

Tuorr, C., "Medecine and the State in Canada: The Extra-Billing Issue in Perspective ", Revue Canadienne de Science Politique, vol. 21, n 2, 1988 : 267-296. 